\title{
Lusioersily
}

\section{Can Corticomuscular Coupling be Useful in Designing Hybrid-Brain Robot Interfaces Towards Hand Functional Recovery?}

Chowdhury, A., Dutta, A., \& Prasad, G. (Accepted/In press). Can Corticomuscular Coupling be Useful in Designing Hybrid-Brain Robot Interfaces Towards Hand Functional Recovery? In Proc. 2019 IEEE International Conference on Systems, Man and Cybernetics (SMC) October 6-9, 2019, Bari, Italy. IEEE.

Link to publication record in Ulster University Research Portal

Published in:

Proc. 2019 IEEE International Conference on Systems, Man and Cybernetics (SMC) October 6-9, 2019, Bari, Italy.

\section{Publication Status:}

Accepted/In press: 23/06/2019

\section{Document Version \\ Author Accepted version}

\section{General rights}

Copyright for the publications made accessible via Ulster University's Research Portal is retained by the author(s) and / or other copyright owners and it is a condition of accessing these publications that users recognise and abide by the legal requirements associated with these rights.

\section{Take down policy}

The Research Portal is Ulster University's institutional repository that provides access to Ulster's research outputs. Every effort has been made to ensure that content in the Research Portal does not infringe any person's rights, or applicable UK laws. If you discover content in the Research Portal that you believe breaches copyright or violates any law, please contact pure-support@ulster.ac.uk. 


\title{
Can Corticomuscular Coupling be Useful in Designing Hybrid-Brain Robot Interfaces Towards Hand Functional Recovery?
}

\author{
Anirban Chowdhury ${ }^{1}$, Ashish Dutta ${ }^{2}$, and Girijesh Prasad ${ }^{1}$
}

\begin{abstract}
Corticomuscular coupling is so far widely explored only for the assessment of motor recovery. However, the possibility of using it for driving hybrid Brain-robot interfaces (h-BRI) is much ignored. Being an important factor for in re-establishing the connection between brain and muscle, corticomuscular coupling might play an important role in providing meaningful neurofeedback of the therapeutic task performance leading to faster recovery. This paper reports a 5-week-long clinical study on 4 chronic stroke patients where we have used correlation of band-limited power time-courses (CBPT), a measure of corticomuscular coupling, as a feature for providing multimodal neurofeedback for hand exoskeleton based therapy. The primary outcome is measured using the standard motor recovery measures of action research arm test (ARAT) and gripstrength (GS). As a secondary measure of recovery, the resting state MEG was also recorded on a weekly basis. The usability of the system was also assessed using the visual-analog-scale measurements of the patients' motivation and fatigue. Results show that the $h$-BRI performance increased significantly (pvalue $<0.05$ ) from the average $58.16 \pm 7.81 \%$ from the beginning to the $77.17 \pm 3.65 \%$ at the end session. A positive change in the ARAT $(+23.75)$ and GS $(+9.83 \mathrm{~kg})$ scores was also observed as compared to the baseline. Both of these changes were beyond the minimal clinically important difference (MCID) limit. Overall, the study shows that corticomuscular coupling can be used to design a high-performing BRI which also has the potential of being clinically effective.
\end{abstract}

\section{INTRODUCTION}

One of the most challenging parts of poststroke neurorehabilitation is the hand functional recovery. The conventional therapies mostly depend on therapist-assisted passive repetitive training which is not so effective in a shorter duration and also less motivating for the patients [1]. This increases the cost of rehabilitation due to the unavailability of expert professionals. Moreover, in the chronic stage, it is, even harder for the patients to regain the functionalities of their hand, which is most essential in carrying out daily living activities [2]. The introduction of robot-assisted therapies is very promising as it reduces the need for an expert therapist, thereby cost-effective, although the engagement of the patients with the therapeutic task is a big challenge as it plays an important part in the recovery process [3].

Therefore researchers in past two decades extensively explored the possibility of using brain-robot interfaces as a

*This work was supported by UK India Education and Research Initiative (UKIERI-DST-2013-14/126 and DST-UKIERI-2016-17-0128).

${ }^{1}$ Anirban Chowdhury and Girijesh Prasad are with School of Computing, Engineering, and Intelligent Systems, Ulster University, Derry Londonderry, BT487JL, UK.

(a. chowdhury@ulster.ac.uk; g.prasad@ulster.ac.uk)

${ }^{2}$ Ashish Dutta is with the Department of Mechanical Engineering, IIT Kanpur, Kanpur, UP 208016, India (adutta@iitk. ac.in) control mode for robotic rehabilitation as it is directly related to the patients' attention and active involvement [4], [5], [6], [7], [8]. However, predicting the patient's motor intent with a higher level of accuracy is very important to give meaningful neurofeedback, otherwise, it will frustrate the patient and could impede motor recovery. But the complexity of the EEG signals, lower signal to noise ratio, and the non-stationary nature of the data itself together pose a huge challenge to achieving satisfactory performance in brainrobot interfaces [9], [10]. People have tried using advanced computational modeling adaptive classification approaches to cater to this problem [11]. Recently, combining other biological signals with the brain-signals became a trend as it can make use of additional modes of physiological control such as heart-rate, eye-tracking, and EMG. This new generation of BRI is called the hybrid (h-BRI) [12].

From the neurorehabilitation perspective, the use of EMG signal along with the EEG signal has great potential for designing advanced BRI systems [13]. This is because of the relation of corticomuscular coupling (the connection between the brain and muscle activations) with the motor recovery [14]. Several studies in the past highlighted this relationship by calculating corticomuscular coherence (CM-Coh) as a measure of corticomuscular coupling which increases as the patients regains their motor functionality [15], [16]. Although CM-Coh provides a good picture for motor recovery, it is not very useful in predicting the motor-intentions on a single trial basis and hence not useful for designing BRI systems [17], [18]. The drawbacks of CM-Coh to be a suitable feature for EEG classifiers are: shorter epochs suffer from less accurate estimates of CM-Coh, the inter-trial inconsistencies, and more importantly it significantly reduced after stroke.

In the quest to develop a new method for corticomuscular coupling which can also act as a feature for driving hBRI systems, we previously introduced correlation of bandlimited power time-courses (CBPT) as a corticomuscular coactivation index capable of single-trial based prediction of motor attempt, based on healthy participants and 8 stroke patients of briefer (mostly 8 months or less) stroke history [19]. We showed that CBPT is able to drive an h-BRI system with a satisfactory level of accuracy: $92.81 \pm 2.09 \%$ for healthy individuals and $84.53 \pm 4.58 \%$ for stroke patients. The CBPT feature also outperformed CM-Coh feature in single-trial based prediction of motor movement and also performed better than conventional EEG based BRI [18]. These findings motivated us to investigate further implications of CBPT in clinical recovery. To this end, we conducted a 5-week-long clinical trial on 4 chronic hemiparetic stroke patients and 
TABLE I

BASELINE DEMOGRAPHICS OF THE PARTICIPANTS

\begin{tabular}{|l|l|l|l|l|l|l|l|l|}
\hline $\begin{array}{l}\text { Participant } \\
\text { Code }\end{array}$ & Sex & $\begin{array}{l}\text { Age } \\
\text { (years) }\end{array}$ & $\begin{array}{l}\text { MMSE Scores } \\
\text { (Total=30) }\end{array}$ & $\begin{array}{l}\text { Impairment } \\
\text { Side }\end{array}$ & $\begin{array}{l}\text { Months Since } \\
\text { Stroke }\end{array}$ & $\begin{array}{l}\text { ARAT } \\
\text { (baseline) }\end{array}$ & $\begin{array}{l}\text { GS (kg) } \\
\text { (baseline) }\end{array}$ & $\begin{array}{l}\text { Stroke } \\
\text { Type }\end{array}$ \\
\hline SECN & F & 60 & 28.5 & Right & 22 & 35 & 3 & Ischemic \\
\hline MNLY & M & 56 & 30 & Left & 28 & 6 & 11.7 & Ischemic \\
\hline ANLD & F & 69 & 28 & Right & 24 & 29 & 13.7 & Ischemic \\
\hline MNGR & F & 65 & 27 & Left & 18 & 24 & 12 & Ischemic \\
\hline JSLY & M & 58 & 28 & Right & 17 & 26 & 13.3 & Ischemic \\
\hline
\end{tabular}

provided them with hand exoskeleton based neurofeedback contingent on their grasp attempt. The prediction of the grasp-attempt is done using the CBPT between the EEG and EMG signals, which was used as a feature for a supportvector-machine based classifier. In this paper, we report the clinical effectiveness of the CBPT based h-BRI intervention related to hand-functional recovery.

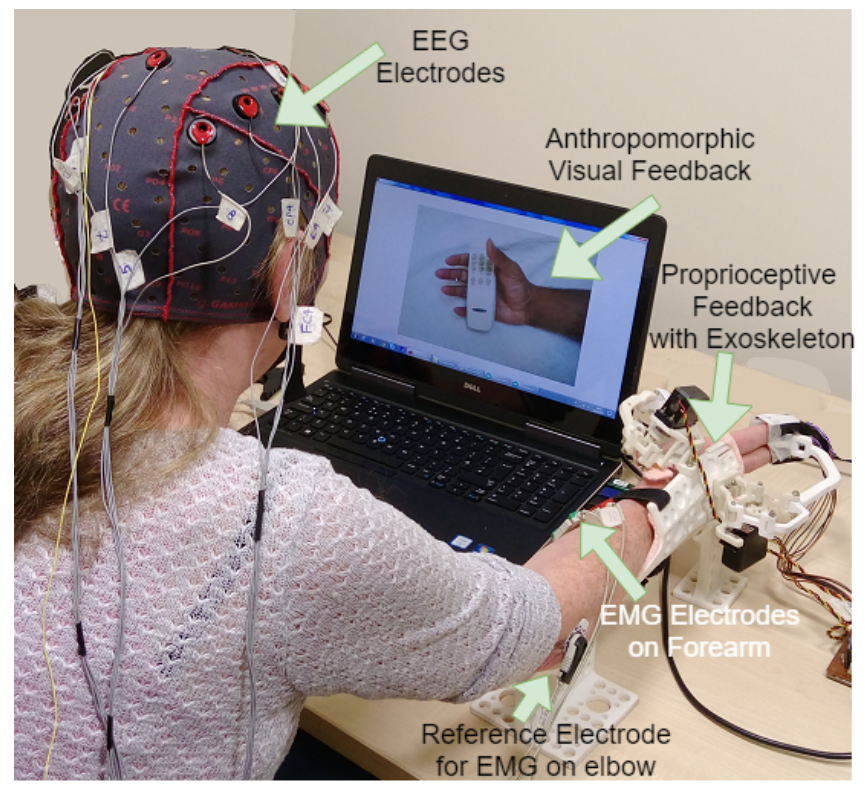

Fig. 1. The experimental set up is showing the EEG and EMG electrode placements while the patient is doing the therapeutic task wearing the exoskeleton in her impaired hand. The visual feedback is given on the computer screen.

\section{MATERIALS AND METHODS}

\section{A. Participants}

From an initial recruitment pool of 15 participants, 5 participants ( 3 female, Table I) were selected based on inclusion and exclusion criteria according to a protocol and signed consent form approved by the ethics committee of Ulster University. The selection was based on the ability of the participants to follow instructions related to the task, as assessed by a score of at least 27 (out of 30 ) on a mini-mental state examination of cognitive impairment, and based on lack of history of epilepsy which might interfere with the signals. The 5 participants were aged 56-69 years, had a chronic
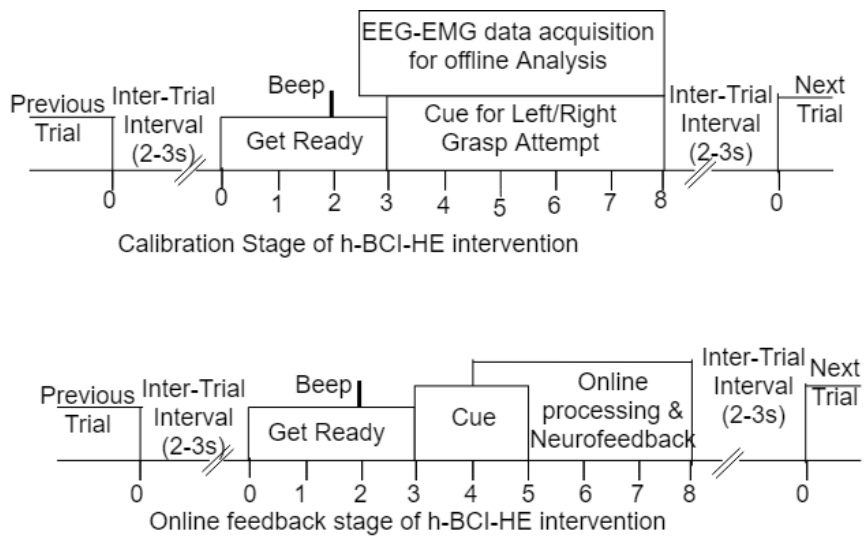

Fig. 2. The timing diagram of a single trial during the calibration (above) and the online feedback (below) phase.

stroke (17-28 months since stroke), and were medically assessed to have negligible chances for auto-recovery. Of the 5 participants, one male participant JSLY dropped out partway through the study (because of hand fracture in an accident not related to the clinical trial) and was not included in the final analyses. All participants suffered from an ischemic stroke but retained some residual EMG activation at the time of recruitment. Of the final 4 participants, 2 were impaired in their left hand, and 2 were impaired in their right hand. The baseline demographics of the participants are shown in Table I.

\section{B. Experimental set-up \& Data Acquisition}

The EEG data were recorded from the following channels according to the 10-20 international systems: F3, FC3, C3, CP3, P3, FCz, CPz, F4, FC4, C4, CP4, and P4. The EMG channels were on the left and right flexordigitorumsuperficialis (FDS) muscles which are responsible for the flexion of the fingers. The standard g.tec ${ }^{\mathrm{TM}}$ amplifier was connected to a custom signal processing and neurofeedback generation software built in the MATLAB/Simulink ${ }^{\mathrm{TM}}$ platform, which also controlled the entire experimental paradigm. The hand exoskeleton hand two arms; one for moving the index and middle fingers in a coupled fashion, and another to drive the thumb. Both arms are controlled by servomotors attached to a four-bar mechanism which facilitates the finger-motion along natural human finger trajecto- 


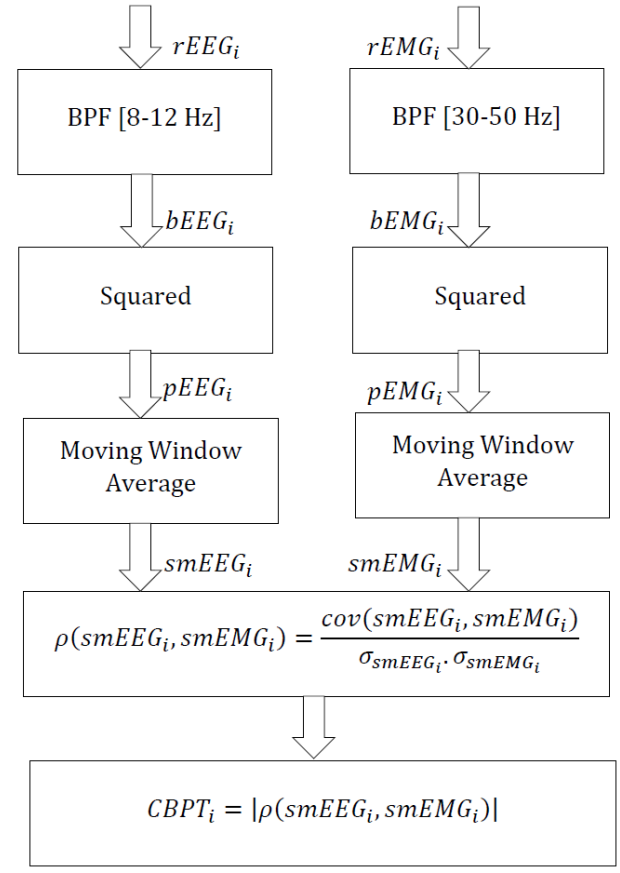

Fig. 3. The block diagram showing the pipeline for calculating the CBPT index from EEG and EMG signals.

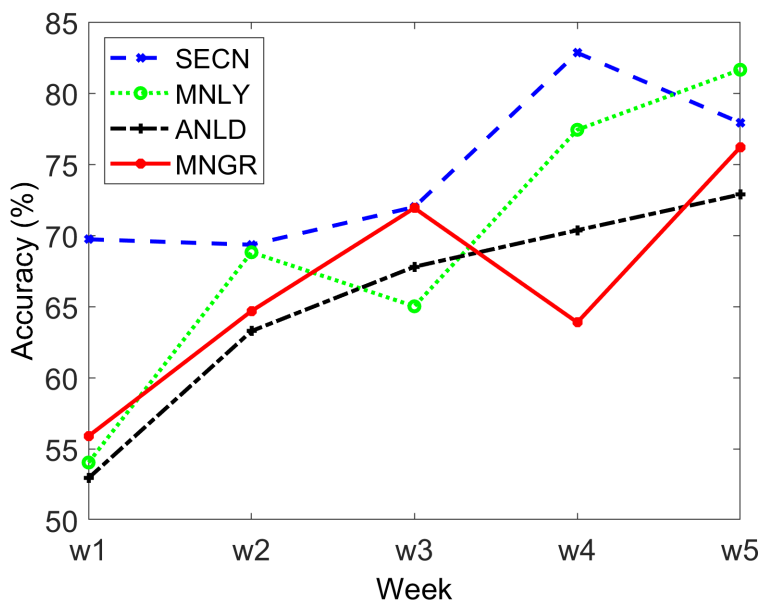

Fig. 4. BRI performance as measured by weekly variation of classification accuracy for all the participants.

ries. An arm-rest is attached to the exoskeleton to support the forearm being in a comfortable position throughout the experiment. The experimental set-up is shown in Fig. 1.

\section{Intervention}

The intervention was composed of a physical practice stage using assist-as-needed control of the exoskeleton by the participant, followed by an h-BRI mediated neurofeedback stage. The h-BRI was calibrated before entering into the online neurofeedback stage. During the calibration, the participants were asked to make a grasp-attempt using the left or right hand according to the cue presented during the trial. A trial during the calibration stage is shown in Fig. 2.

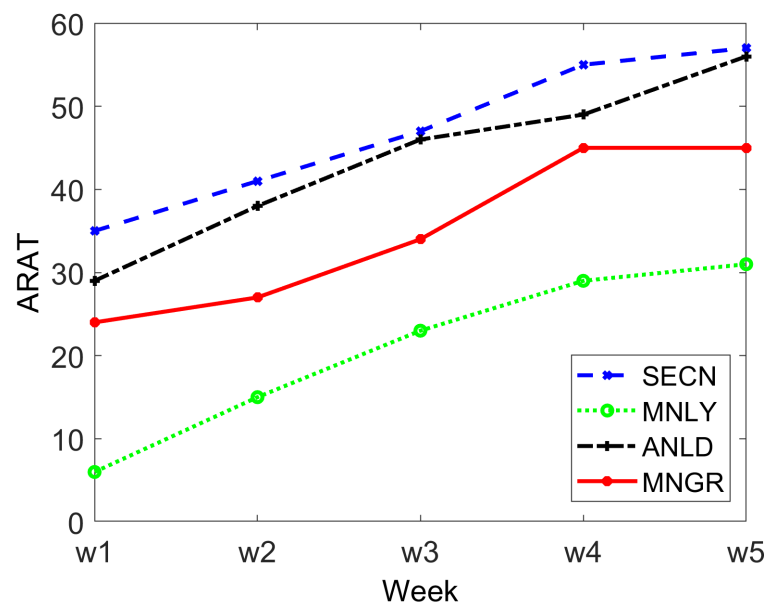

Fig. 5. Weekly variations in ARAT for all the participants.

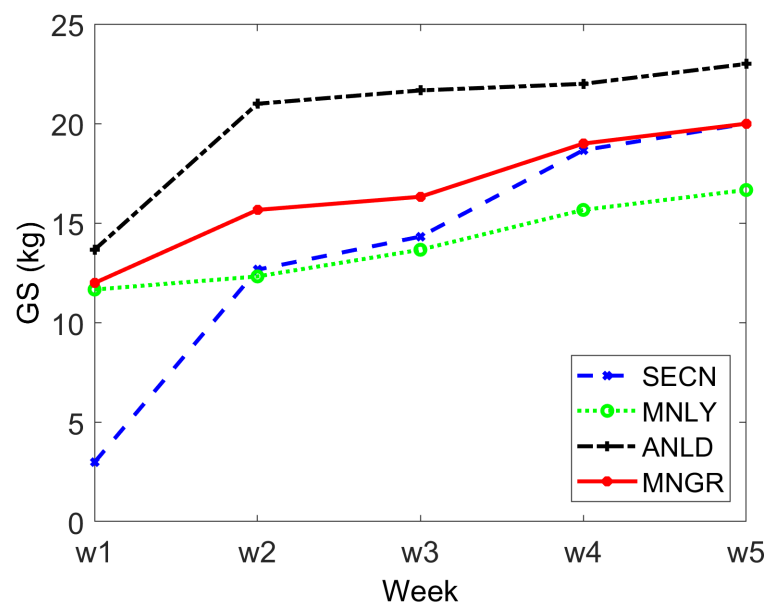

Fig. 6. Weekly variations in GS for all the participants.

At the start of the trial, there was a 'get ready' warning message, followed 2 seconds later by a warning beep, then followed 1 second later by a cue in the form of a left or right hand appearing on the screen. During the remaining 5 sec of the trial, the EEG and EMG data were acquired while the participant tried to make a grasp. The inter-trial interval was $2-3 \mathrm{sec}$. There were 40 such trials in a calibration run, and the entire calibration phase was composed of two such runs. These EEG and EMG data, along with the appropriate class labels (class1: left-hand grasp, and class2:right-hand grasp) were then used to extract the CBPT features (a full description of extracting the CBPT features can be found in our previous work [19]) which were then used to train an support-vector-machine (SVM) classifier with a linear kernel. The same classifier was then used to predict the graspattempt at the online neurofeedback stage and the participants received multimodal neurofeedback which is composed of 1) visual feedback of a virtual hand grasping a TV-remote, and 2) synchronized to the visual feedback the exoskeleton moved the fingers to complete the grasp in order to provide 
proprioception of the same motion.

\section{CBPT feature extraction and Classification}

A detailed description, rationale and performance of CBPT method as a corticomuscular coactivation index capable of single-trial based prediction of motor-attempt has already been introduced in our previously published work [19]. Here we briefly describe the CBPT method to highlight how the EEG and EMG signals are fused to build an h-BRI feature. The block diagram showing the pipeline for calculating the CBPT index is shown in Fig. 3. At the first stage, the raw EEG $\left(r E E G_{i}\right)$ and EMG $\left(r E M G_{i}\right)$ data from trial $i$ are bandpass filtered to their respective frequency bands (8-12 $\mathrm{Hz}$ for EEG and $30-50 \mathrm{~Hz}$ for EMG). Then the bandpass filtered EEG and EMG $\left(b E M G_{i}\right)$ and EMG $\left(b E M G_{i}\right)$ are then squared to get the EEG and EMG band-powers $\left(p E M G_{i}\right)$ and EMG $\left(p E M G_{i}\right)$. This bandpower EEG and EMG are then moving window averaged with a smoothing window of $1 \mathrm{~s}$ for EEG $\left(s m E E G_{i}\right)$ and $32 \mathrm{~ms}\left(s m E M G_{i}\right)$ for EMG. The length of the smoothing window is obtained empirically for optimal performance of the classifier. Finally, a suitable time window of [3.5:5] s (i.e. $+0.5 \mathrm{~s}$ to $2 \mathrm{~s}$ after the cue) was chosen from $s m E E G_{i}$ and $s m E M G_{i}$ to calculate the Pearsons correlation between these two time-courses. The absolute value of this correlation above the chance level is considered as the CBPT index for that trial $\left(C B P T_{i}\right)$. We have used the corrcoef function of MATLAB for calculating the correlation coefficient and its $p$-value. If the $p$-value is greater than $0.05, C B P T_{i}$ valued is not considered and replaced by zero. A feature vector comprised of $C B P T_{i}$ from all the different EEG and EMG channel combinations was formed for every trial within the calibration stage and then fed into the SVM model for classifier generation. At the online feedback stage, the same CBPT feature extraction process is repeated and classified by the SVM classifier generated at the calibration stage.

\section{E. Outcome measures}

The outcome of the study is measured using the standard motor outcome measures such as GS and ARAT. The GS was measured using a dynamometer on which the participant had to apply grip-force three times and the average of them constitutes a single measurement in $\mathrm{kg}$. The ARAT is the measure of different functionalities of a human hand such as grasp, grip, pinch, and gross movements. It is measured in a scale between $0-56$, where 0 indicates full disability and 56 indicates achieving full functionality of the hand. The GS and ARAT measurements were taken on a weekly basis.

\section{RESUlTs \& Discussion}

We evaluated the performance of the h-BRI on the basis of its classification accuracy (CA) in controlling the handexoskeleton device. The change in the $\mathrm{CA}$ is depicted in Fig. 4. The largest improvement in the CA was found for participant MNLY, as it increased $27.61 \%$ from the first session $(54.04 \%)$ to the last session $(81.65 \%)$. The smallest change in CA was observed for SECN, as it increased $8.20 \%$ from the first session (69.73\%) to the last session $(77.93 \%)$. The group-mean increase in CA was $19.01 \%$, which was also statistically significant ( $p$-value $<0.05$, paired t-test). The average CA in the first week was $58.16 \pm 7.81 \%$, which was lower than the satisfactory performance level of $70 \%$ often prescribed in the BRI literature [20]. The fact that at the last session the average CA increased up to $77.17 \pm 3.65 \%$ shows that CBPT based h-BRI have a positive impact on the controllability of a robotic device by stroke patients. As we know, better control of the therapeutic device leads to higher motivation level for the patients, which in turn helps them engage more with the task.

The clinical outcome, measured by GS and ARAT, also showed significant ( $p$-value $<0.05$, paired t-test) improvements in hand functionality, as the group-mean change in GS, was found to be $+9.83 \mathrm{~kg}$ and the group-mean change in ARAT score was +23.75. The results of ARAT and GS variations are shown in Fig. 5 and Fig. 6 respectively. The highest change in GS was found in SECN $(+17 \mathrm{~kg})$ and the lowest was in MNLY, which was $5 \mathrm{~kg}$. The highest change in ARAT was found in ANLD (+27 kg) and the lowest was in MNGR, $(+21 \mathrm{~kg})$. The average change in GS and ARAT were also greater than the minimal clinically important difference (MCID) limit, which is $6.2 \mathrm{~kg}$ and 5 for the GS and ARAT respectively [21]. Although the study lacked the control group to fully justify the clinical effectiveness of the intervention, these clinically important changes in the hand functional recovery measures shows that the CBPT based h-BRI have a positive impact on the motor recovery in chronic stroke. In fact, the functional recovery was stopped for a long time for these patients and hence the impact of the applied intervention can be considered to be the most reliable cause of re-initiating the recovery process.

Further, We investigated the neurophysiological effect of the intervention by looking at the changes in beta bandpower, which is widely used as a biomarker for motor recovery. The topoplots for the first and last session of the h-BRI based intervention are plotted in Fig. 7. The topoplots are generated by calculating the average beta band event-relateddesynchronization/synchronization (ERD/ERS) for EEG every channel. Results show significant $(p-$ value $<0.05$, paired t-test) reduction in both the ipsi- and contra-lesional betadesynchronization for all the participants. For example, in Fig. 7(a), for SECN most significant $(p$-value $<0.05)$ changes in beta-desynchronization were found in $\mathrm{C} 3, \mathrm{CP} 3$, $\mathrm{FCz}, \mathrm{FC} 4, \mathrm{C} 4$, and CP4 channels. Similar changes can also be observed for ANLD (Fig. 7(c)), where the most significant $(p$-value $<0.05)$ beta-desynchronization changes occurred in F3, FCz, F4, FC4, C4, and CP4. In MNLY (Fig. 7(b)) most significant $(p-$ value $<0.05)$ changes were found in FC3 and $\mathrm{FCz}$, while in MNGR (Fig. 7(d)) it was FCz, CP3, F4, C4, and CP4.

\section{CONCLUSIONS}

The paper explores the answer to the question of whether a corticomuscular coupling based h-BRI system is possible to apply for hand rehabilitation for chronic stroke patients. 
SECN

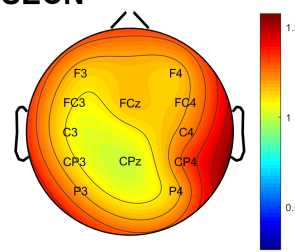

First Session

(a)

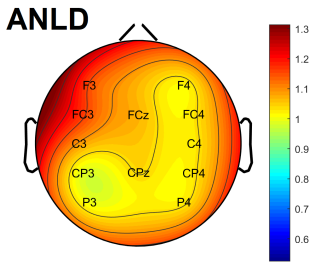

First Session

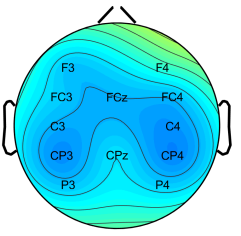

Last Session

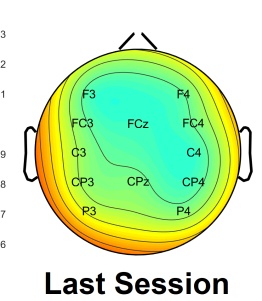

(c)

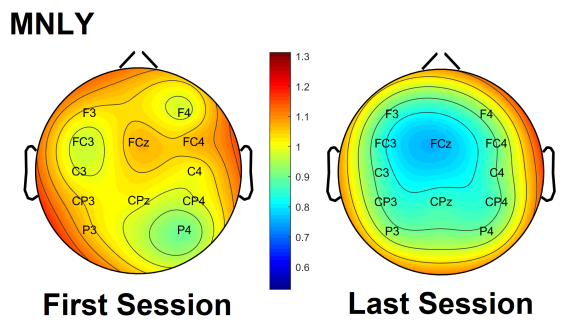

(b)

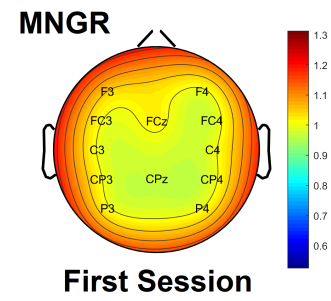

(d)

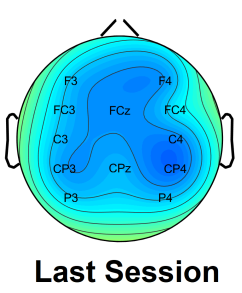

Last Session

Fig. 7. Beta-band ERD variations of all the participants: (a) SECN, (b) MNLY, (c) ANLD, (d) MNGR

Results show that CBPT based measure of corticomuscular coupling can be used as a feature which can lead to highperforming h-BRI system as the classification accuracy is seen to be improved throughout the therapeutic sessions. The CBPT based h-BRI intervention also led to a significant positive change in motor outcome measures beyond the MCID limit, which indicates its potential for effective motor recovery. The neurophysiological changes, measured by beta band-power change, also supported the motor recovery outcome which further reinforces the feasibility of the CBPT based h-BRI system for hand functional recovery.

\section{ACKNOWLEDGMENT}

We thank Ms. Annmarie Kelly, Occupational Therapist, Altnagelvin Hospital, Derry Londonderry and Dr. Yogesh Kumar Meena for their help in recruiting the stroke patients and helping with the data collection.

\section{REFERENCES}

[1] X. Zhang, A. M. Elnady, B. K. Randhawa, L. A. Boyd, and C. Menon, "Combining mental training and physical training with goal-oriented protocols in stroke rehabilitation: A feasibility case study," Frontiers in Human Neuroscience, vol. 12, p. 125, 2018.

[2] A. Chowdhury, Y. K. Meena, H. Raza, B. Bhushan, A. K. Uttam, N. Pandey, A. A. Hashmi, A. Bajpai, A. Dutta, and G. Prasad, "Active physical practice followed by mental practice using BCI-driven hand exoskeleton: A pilot trial for clinical effectiveness and usability," IEEE Journal of Biomedical and Health Informatics, vol. 22, no. 6, pp. 1786-1795, Nov 2018.

[3] G. Prasad, P. Herman, D. Coyle, S. McDonough, and J. Crosbie, "Applying a brain-computer interface to support motor imagery practice in people with stroke for upper limb recovery: a feasibility study." Journal of neuroengineering and rehabilitation, vol. 7, no. 1, p. 60, 2010.

[4] S. E. Fasoli, H. I. Krebs, J. Stein, W. R. Frontera, R. Hughes, and N. Hogan, "Robotic therapy for chronic motor impairments after stroke: follow-up results," Archives of Physical Medicine and Rehabilitation, vol. 85, no. 7, pp. 1106-1111, 2004.

[5] E. Buch, C. Weber, L. G. Cohen, C. Braun, M. A. Dimyan, T. Ard, J. Mellinger, A. Caria, S. Soekadar, A. Fourkas, and N. Birbaumer, "Think to move: A neuromagnetic brain-computer interface (BCI) system for chronic stroke," Stroke, vol. 39, no. 3, pp. 910-917, 2008.
[6] A. Ramos-Murguialday, M. Schürholz, V. Caggiano, M. Wildgruber, A. Caria, E. M. Hammer, S. Halder, and N. Birbaumer, "Proprioceptive Feedback and Brain Computer Interface (BCI) Based Neuroprostheses," PLoS ONE, vol. 7, no. 10, 2012.

[7] G. Morone, I. Pisotta, F. Pichiorri, S. Kleih, S. Paolucci, M. Molinari, F. Cincotti, A. Kübler, and D. Mattia, "Proof of Principle of a BrainComputer Interface Approach to Support Poststroke Arm Rehabilitation in Hospitalized Patients: Design, Acceptability, and Usability," Archives of Physical Medicine and Rehabilitation, vol. 96, no. 3, pp. S71-S78, 2015.

[8] A. Chowdhury, S. S. Nishad, Y. K. Meena, A. Dutta, and G. Prasad, "Hand-exoskeleton assisted progressive neurorehabilitation using impedance adaptation based challenge level adjustment method," IEEE Transactions on Haptics, vol. 12, no. 2, pp. 128-140, April 2019.

[9] H. Raza, H. Cecotti, Y. Li, and G. Prasad, "Adaptive learning with covariate shift-detection for motor imagery-based brain-computer interface," Soft Computing, vol. 20, no. 8, pp. 3085-3096, Aug 2016.

[10] S. Roy, D. Rathee, K. McCreadie, and G. Prasad, "Channel selection improves meg-based brain-computer interface," in 2019 9th International IEEE/EMBS Conference on Neural Engineering (NER), March 2019, pp. 295-298.

[11] A. Chowdhury, H. Raza, Y. K. Meena, A. Dutta, and G. Prasad, "Online covariate shift detection-based adaptive braincomputer interface to trigger hand exoskeleton feedback for neuro-rehabilitation," IEEE Transactions on Cognitive and Developmental Systems, vol. 10, no. 4, pp. 1070-1080, Dec 2018.

[12] G. Muller-Putz et al., "Towards Noninvasive Hybrid BrainComputer Interfaces: Framework, Practice, Clinical Application, and Beyond," Proceedings of the IEEE, vol. 103, no. 6, pp. 926-943, 2015.

[13] R. Leeb, H. Sagha, R. Chavarriaga, and J. D. R. Millán, "A hybrid brain-computer interface based on the fusion of electroencephalographic and electromyographic activities," Journal of Neural Engineering, vol. 8, no. 2, p. 025011, 2011.

[14] K. von Carlowitz-Ghori, Z. Bayraktaroglu, F. U. Hohlefeld, F. Losch, G. Curio, and V. V. Nikulin, "Corticomuscular coherence in acute and chronic stroke," Clinical Neurophysiology, vol. 125, no. 6, pp. 11821191, 2014.

[15] P. Belardinelli, L. Laer, E. Ortiz, C. Braun, and A. Gharabaghi, "Plasticity of premotor cortico-muscular coherence in severely impaired stroke patients with hand paralysis," NeuroImage: Clinical, vol. 14, pp. 726-733, 2017.

[16] R. Krauth, J. Schwertner, S. Vogt, S. Lindquist, M. Sailer, A. Sickert, J. Lamprecht, S. Perdikis, T. Corbet, J. d. R. Millán, H. Hinrichs, H.J. Heinze, and C. M. Sweeney-Reed, "Cortico-Muscular Coherence Is Reduced Acutely Post-stroke and Increases Bilaterally During Motor Recovery: A Pilot Study," Frontiers in Neurology, vol. 10, February 2019. 
[17] A. Chwodhury, H. Raza, A. Dutta, S. S. Nishad, A. Saxena, and G. Prasad, "A study on cortico-muscular coupling in finger motions for exoskeleton assisted neuro-rehabilitation," in 2015 37th Annиal International Conference of the IEEE Engineering in Medicine and Biology Society (EMBC), Aug 2015, pp. 4610-4614.

[18] A. Chowdhury, H. Raza, A. Dutta, and G. Prasad, "EEG-EMG based hybrid brain computer interface for triggering hand exoskeleton for neuro-rehabilitation," in Proceedings of the Advances in Robotics, ser. AIR '17. New York, NY, USA: ACM, 2017, pp. 45:1-45:6.

[19] A. Chowdhury, H. Raza, Y. K. Meena, A. Dutta, and G. Prasad, "An EEG-EMG correlation-based brain-computer interface for hand orthosis supported neuro-rehabilitation," Journal of Neuroscience Methods, vol. 312, pp. $1-11,2019$.

[20] A. Kbler, N. Neumann, B. Wilhelm, T. Hinterberger, and N. Birbaumer, "Predictability of brain-computer communication," Journal of Psychophysiology, vol. 18, no. 2/3, pp. 121-129, 2004.

[21] D. Rathee, A. Chowdhury, Y. K. Meena, A. Dutta, S. McDonough, and G. Prasad, "Brainmachine interface-driven post-stroke upperlimb functional recovery correlates with beta-band mediated cortical networks," IEEE Transactions on Neural Systems and Rehabilitation Engineering, vol. 27, no. 5, pp. 1020-1031, May 2019. 